\title{
Volumetric error modelling of a stereo vision system for error correction in photogrammetric three-dimensional coordinate metrology
}

\author{
Mohammed A. Isa ${ }^{1}$, Danny Sims-Waterhouse ${ }^{1,2}$, Samanta Piano ${ }^{1}$ and Richard Leach ${ }^{1,2}$ \\ ${ }^{1}$ Manufacturing Metrology Team, Faculty of Engineering, University of Nottingham, UK \\ ${ }^{2}$ Taraz Metrology, Nottingham, UK
}

\begin{abstract}
Optical three-dimensional coordinate measurement using stereo vision has systematic errors that affect measurement quality. This paper presents a scheme for measuring, modelling and correcting these errors. The position and orientation of a linear stage are measured with a laser interferometer while a stereo vision system tracks target points on the moving stage. With reference to the higher accuracy laser interferometer measurement, the displacement errors of the tracked points are evaluated. Regression using a neural network is used to generate a volumetric error model from the evaluated displacement errors. The regression model is shown to outperform other interpolation methods. The volumetric error model is validated by correcting the three-dimensional coordinates of the point cloud from a photogrammetry instrument that uses the stereo vision system. The corrected points from the measurement of a calibrated spherical artefact are shown to have size and form errors of less than $50 \mu \mathrm{m}$ and $110 \mu \mathrm{m}$ respectively. A reduction of up to $30 \%$ in the magnitude of the probing size error is observed after error correction is applied.

Keywords: Stereo vision, Photogrammetry, Volumetric error, Error correction, Laser interferometry
\end{abstract}

\section{Introduction}

The process of reconstructing a three-dimensional (3D) coordinate measurement from a series of twodimensional images is complex and can result in significant systematic errors [1]. Hence, active optical 3D methods use an additional laser or light source to aid the reconstruction of 3D shape, while passive methods employ visual cues, such as texture, shading, focus and silhouettes. The performance and cost of these methods depends on the measurement technique on which they are based. The measurement techniques can be divided into triangulation, time-of-flight and interferometry [2]. While methods based on time-of-flight and interferometry require industrial-level sensors and instrumentation, triangulation-based systems can be built from readily available components [3]. In application, triangulation-based systems usually consist of a camera combined with one or more of a laser, structured light source or additional cameras. It is common to use two cameras (stereo vision) in triangulation methods, such as laser triangulation, fringe projection and photogrammetry for higher accuracy [4-6].

The stereoscopic principle lies at the heart of various optical 3D measurements, autonomous navigation, medical imaging, tracking and augmented reality [7]. Despite its widespread use, stereo vision suffers from systematic errors that affect the accuracy of the 3D reconstruction processes. Due to the dependency on multiple parameters, such as focal length, distortion coefficients, pixel quantisation, focus and more, it is difficult to accurately map and correct for reconstruction errors [8, 9]. Rigorous uncertainty analyses of stereo and multi-view 3D measurements using Monte-Carlo simulation have demonstrated that there is significant systematic error behaviour $[1,10,11]$. The deviation from the known motion of detected target points has been used to obtain a statistical measure of systematic error [7]. The tracking of a known motion trajectory has also been used in 
geometric characterisation of optical 3D systems $[12,13]$. While deviations from a known trajectory can give an insight into the magnitude of the systematic error, they lack a traceable reference for dimensional measurement. Therefore, the use of a laser interferometer for simultaneous calibration of the displacement of a moving system could provide traceability through the wavelength of the light source. In addition, instantaneous tracking minimises the effect of the motion system and environment on the magnitude of the error.

Interferometers measure length with direct traceability through the calibrated wavelength of their optical light source [14]. Adoption of laser interferometers is widespread for measurement of geometric errors of machines with sub-micrometre accuracy $[15,16]$. Laser interferometers have been used to study the volumetric map of errors in machine tools $[17,18]$ and coordinate measurement machines (CMMs) $[19,20]$; however, there is lack of research in the area of volumetric error modelling for optical 3D systems. Echerfaoui et al. [21] used a laser interferometer to estimate the kinematic error components of a $\mathrm{CMM}$ and generated a volumetric error map using a neural network. Bräuer et al. [22] used discrete systematic error estimation from the error of length of a ball bar at different 3D measurement locations. Challenges in automated gathering and modelling of the error data hindered error compensation to validate the systematic error. As a result, the need for an alternative length measurement strategy was acknowledged.

This paper presents a method for evaluating the discrete spatial errors of target points measured by stereo vision with reference to an interferometry tracking system. By tracking the linear and angular position of a linear stage using a laser interferometer, the displacements of the optical target points, placed on the same stage, are used to evaluate the error in length measurement of the stereo vision system. To facilitate error estimation at any position within the stereo-camera measurement volume, the volumetric error is modelled using neural network regression. Therefore, using the regression model, this approach allows compensation for errors in 3D points obtained by any stereoscopic triangulation method.

Photogrammetry is applied in this paper for the purpose of testing the error correction approach on an object in place of the optical target used for the error evaluation process. By using a reference artefact of calibrated size and form, the stereo images of the object's surface are used for photogrammetric reconstruction. The compact nature of photogrammetric systems makes them flexible and integrable into machine tools [23], enhancing their in-situ and in-process applications [24]. Also, it has been shown that the surface texture of parts fabricated by additive manufacturing can be used as the source of disparity for feature extraction and matching [25]. There are various commercial and open-source software packages for carrying out computations in photogrammetry; tools from OpenMVG are applied in this work. OpenMVG is an open-source collection of multi-view geometry libraries that can be used for 3D reconstruction using a structure-from-motion(SfM) pipeline [26]. Stereo image pair correspondences obtained by matching image features are triangulated and grouped into sets of multi-view observations of consistent features visible from all viewpoints in a set [27]. The use of triplets of image views rather than just doublets can significantly improve computational robustness; for instance, a more stable calculation of camera location is obtained using a method that uses a trifocal tensor as opposed to the more common essential matrix in OpenMVG [28]. Due to the susceptibility of the reconstruction process in photogrammetry to outliers in image data and feature correspondences [29], automated object identification and segmentation from the reconstructed point cloud data can be challenging [30]. Hence, in this paper, additional constraints and filters are used to preclude manual intervention and improve measurement quality. 
The displacement measurement and photogrammetry experiments are described in Section 2, along with the calibration process for the artefact. Section 3 covers the computational procedures used to evaluate and model the errors from the displacement measurement experiment. Displacement errors observed by the stereo vision system are computed along the six degrees of freedom of the optical target. In addition, using laser interferometer measurements as the traceable reference, the discrete volumetric error of the stereo vision system is estimated. Section 3 presents regression modelling of the volumetric error using a neural network. An implementation of the volumetric error model in photogrammetric error correction is covered in Section 4, which also discusses the necessary conditions and constraints applied to the photogrammetry pipeline. Finally, results of the displacement tracking and the photogrammetry measurements are presented in Section 5.

\section{Experiment}

Two separate procedures have been used for this research. The displacement measurement procedure is used to obtain information about the errors in the stereo vision system with reference to the laser interferometer. Then, the photogrammetric measurement of an artefact is used to validate the quality of 3D coordinate measurements.

\subsection{Displacement measurement}

The displacements along the linear and angular degrees of freedom of a motorised linear stage were measured for the $100 \mathrm{~mm}$ travel range of the stage. An optical target (a checkerboard pattern $48 \mathrm{~mm}$ $\times 44 \mathrm{~mm}$ ) was used for tracking points on the stage. As shown in Figure 1a), the optical target and a retroreflector were mounted on the moving stage to provide positional feedback to the stereo-camera and the interferometer respectively.

a)

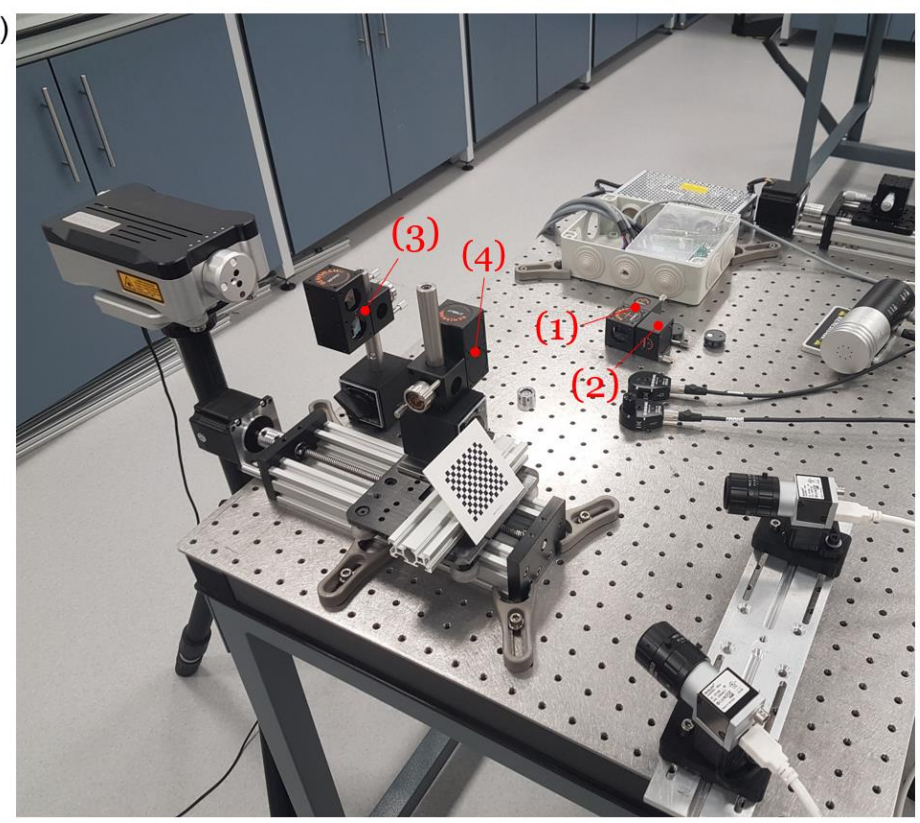

b) Laser interferometer setup for linear measurement

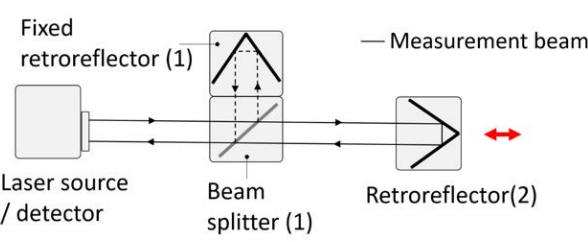

C) Laser interferometer setup for angular measurement

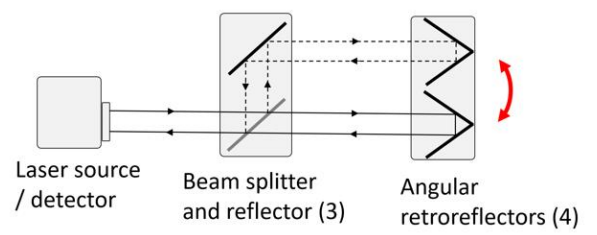

Figure 1. Linear and angular displacement measurement showing a) setup of stereo-camera system and laser interferometer for tracking a motorised linear stage where the labelled optical components are: (1) a beam splitter attached to a fixed retroreflector, (2) a mobile retroreflector, (3) a component 
for beam splitting and reflecting, and (4) a double retroreflector component, b) schematic diagram of laser interferometer aligned for linear measurement, and c) schematic diagram of laser interferometer aligned for angular measurement.

A Renishaw XL-80 laser interferometer system was used to validate the stereo vision measurements of the linear and angular degrees of freedom of the stage. To ensure accurate measurement in varying environmental conditions, the interferometer system is coupled with sensors that measure environmental condition for wavelength compensation. The interferometer is certified by the manufacturer to provide linear measurements with $\pm 0.2 \mu \mathrm{m}$ accuracy within the $100 \mathrm{~mm}$ working range of this application. For angular measurements in the same working range, the laser interferometer's accuracy specification is of the order of $1 \mu$ rad. Figure $1 \mathrm{~b}$ ) shows the optical setup of the beam splitters and reflectors used in the linear displacement measurements. By using the angular type of beam splitter and retroreflector illustrated in Figure 1c), the laser interferometer can be used to measure angular displacements. The optoelectronic detector of the interferometry setup uses the path difference between the measurement and reference beams to determine the linear (or angular) displacement of a moving reflector.

The stereo vision system is composed of two CMOS machine vision cameras with sensors of $1.85 \mu \mathrm{m}$ pixel size and 12.2 megapixels pixel density. Two imaging lenses with $16 \mathrm{~mm}$ focal length were fitted to the cameras. The cameras were mounted together on a stereo-camera rig with an adjustable baseline length and angle. Using the baseline length of $174.5 \mathrm{~mm}$ and an angle of around $36^{\circ}$ between the principal axes of the two cameras, the field of view and focal length of the cameras were set to cover the travel distance of the stage. The use of fixed two-camera system is a common solution to a critical scaling issue, where the baseline length is used to relate photo-scale and metric scale [31]. In fact, among existing passive methods that use cues to reconstruct objects from images, stereo correspondence is the most successful taking into account robustness and number of applications [32]. From detection of analogous feature locations in stereo images, physical 3D points can be triangulated given the intrinsic and geometric parameters of the stereo vision system. The intrinsic parameters (the focal lengths and the principal point locations of the cameras) and the geometric parameters (the relative positions and orientations of the cameras) are determined by the popular Zhang's method [33]. Because this method and most of industrial off-line self-calibration of cameras rely on the accuracy of the features detected [10], optical targets with precise patterns are employed for computation of the imaging parameters.

The output of this experiment consists of scalar values from the interferometric linear distance, angular yaw and pitch angles, and position vectors of the target points obtained from the triangulation of the image points observed by the stereo vision system.

\subsection{Photogrammetry}

A photogrammetry setup consisting of the aforementioned stereo vision system and a rotary stage was prepared for measurement of 3D objects [34]. The same setup of the cameras described in section 2.1 was used. The photogrammetry system captures images of an object as it is rotated on the rotary stage, which has a unidirectional repeatability of $0.007^{\circ}$.

For an artefact to be considered a reference object, it is necessary to calibrate it using a more accurate instrument. A spherical artefact was manufactured from a stainless-steel ball that was spray-painted to have a matt black background and contrasting random white patches as shown in Figure 2. After a thin layer of black paint was sprayed and left to dry, wet smudges of paint from an equally thin layer 
of white spray paint were applied. The surface of the painted spherical artefact was calibrated using a CMM with a $2 \mathrm{~mm}$ diameter stylus. It is recommended to use $\sqrt{\mathrm{Nh} / 2 \pi r}$ parallel circular paths to sample $N$ points on a spherical segment of radius $r$ and enclosed by two parallel planes $h$ distance apart [35]. This approach ensures an approximately uniform distribution of points. Using 1200 points, the recommended distribution of points is used to measure the spherical segment shown in Figure $2 b$ ), resulting in a calibrated radius of $14.976 \mathrm{~mm} \pm 0.032 \mathrm{~mm}$. The radial uncertainty of $\pm 0.032 \mathrm{~mm}$ is expanded from the maximum deviation of $18 \mu \mathrm{m}$ from the best-fit sphere of the points shown in the lower part of Figure $2 \mathrm{~b}$ ). A high confidence level of $99.7 \%$ (coverage factor=3) is adopted for the radial uncertainty because the number of points measured is less than the number of points expected from photogrammetry. The measured radius and radial deviations characterise the size and form variation of the artefact respectively.

a)

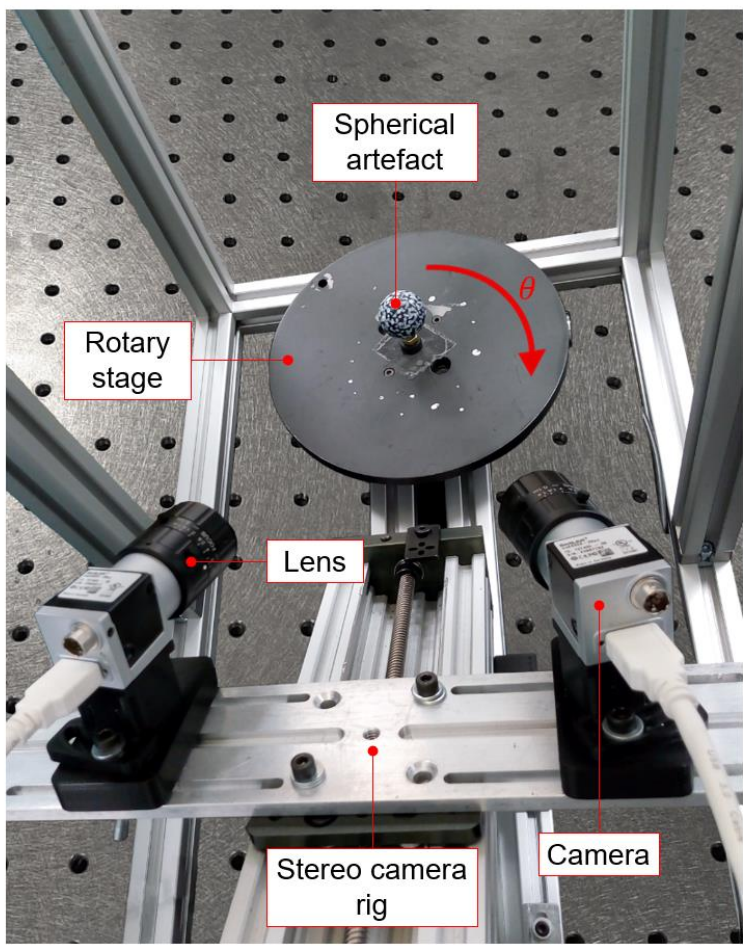

b)

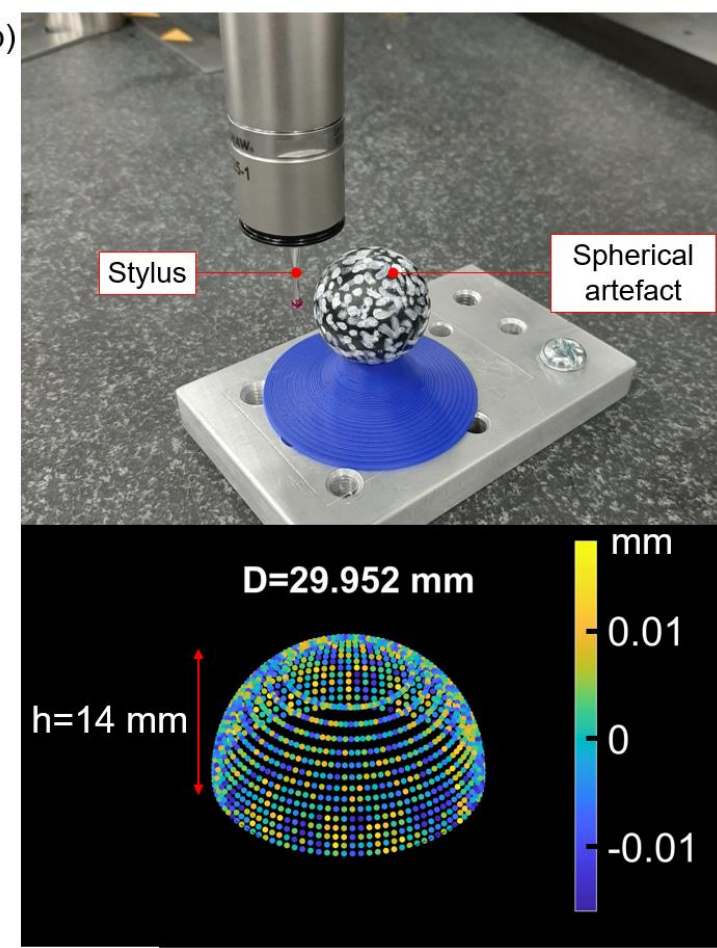

Figure 2. a) Photogrammetry setup showing artefact on a rotary stage. b) Artefact calibration with the $\mathrm{CMM}$ using recommended probing positions on the spherical surface. Radial deviations of the measured points from a fitting sphere are also shown in (b).

\section{Displacement error evaluation and modelling}

The measured displacement values from the two measurement systems are not homogenous because the laser interferometer gives displacement readings along the stage motion axis from the initial stage position while the stereo vision outputs the positions of target points measured from the stereocamera's perspective. Therefore, measurement values from one system should be transformed to an analogous equivalent of the other for comparison and implementation of the measurements. 


\subsection{Kinematic error model}

Due to the imperfections in the components of mechanical systems and the relative motion between them, there are geometric and kinematic errors that affect their performances [36]. These imperfections can be caused by manufacturing defects, temperature changes, dynamic effects, etc. Kinematic errors can be propagated from one moving component to another along both constrained and unconstrained degrees of freedom of the components.

For an arbitrary motion system, Eman et. al. [37] developed a generalised error model based on homogenous transformation matrices to account for error in kinematics. This approach is compatible with the Denavit and Hardenberg (D-H) framework [38] commonly used in multi-body kinematics and robotics. This paper applies the kinematic error model proposed by Eman et al. in evaluating the motion of the linear stage shown in Figure 3a). The error-inclusive position and orientation of the stage can be represented by the homogenous transformation matrix given by

$\boldsymbol{T}(x)=\left[\begin{array}{cccc}1 & -\epsilon_{z} & \epsilon_{y} & x+\delta_{x} \\ \epsilon_{z} & 1 & -\epsilon_{x} & \delta_{y} \\ -\epsilon_{y} & \epsilon_{x} & 1 & \delta_{z} \\ 0 & 0 & 0 & 1\end{array}\right]$

where $x$ is the displacement of the stage in the actuated $x$-direction and the displacement error components in the six degrees of freedom of the stage are given by the three linear components $\left[\delta_{x}, \delta_{y}, \delta_{z}\right]^{T}$ and the three angular components $\left[\epsilon_{x}, \epsilon_{y}, \epsilon_{z}\right]^{T}$. The directions of the error components are shown in the moving coordinate frame $S$ of the moving stage. It is worth noting that the error components in Equation (1) are derived from a first order estimation and depend on the active joint variable $x$. 
a)

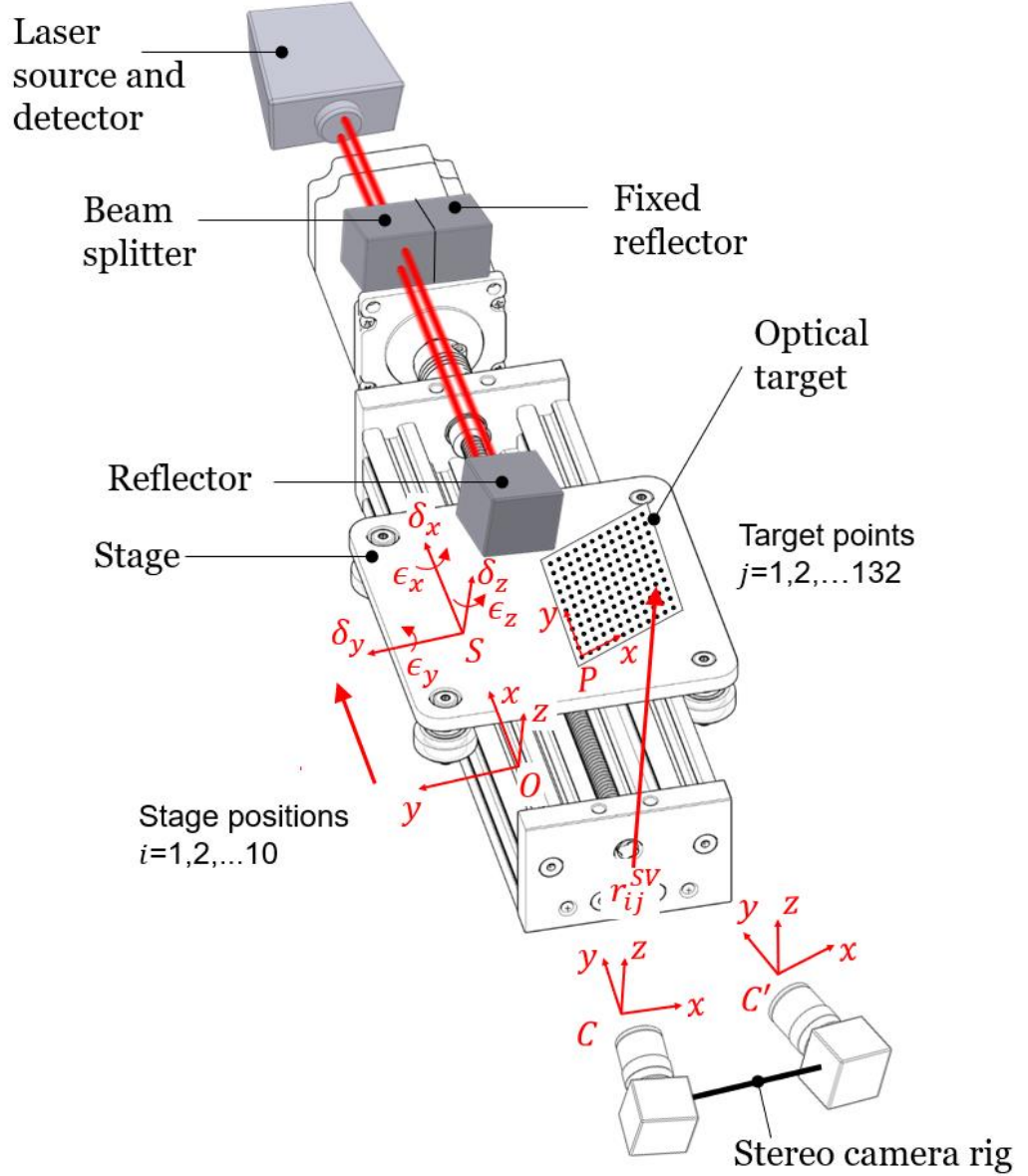

b)

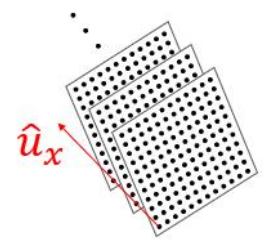

c)

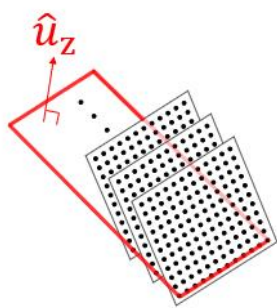

d)

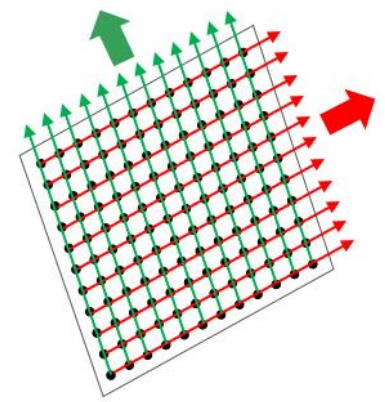

Figure 3. a) Illustration of the coordinate frames and components used in displacement measurement. Evaluation of the b) stage motion axis ( $x$-direction), c) stage's $z$-direction and d) orientation of target plane ( $x y$ plane of frame $P$ ) from the stereo-camera.

\subsection{Displacement error evaluation of the linear stage}

The reference displacement of the stage in the linear, yaw and pitch degrees of freedom are obtained directly from the laser interferometer readings (see section 2.1). Figure 3a) shows the relevant coordinate frames of the stereo-camera $C$, the initial stage position $O$, the optical target frame $P$ and the moving stage frame $S$. Having fixed the interferometric reflector and optical target to the stage, beginning from the point where $S$ coincides with stage's reference frame $O$, the laser interferometer displacement $x_{i}^{L I}$ and stereo vision positions of all $j^{\text {th }}$ target points ${ }^{C} \boldsymbol{r}_{i j}^{S V}$, are measured at stage positions $i=1,2, \ldots, 10$. The target position vectors ${ }^{C} \boldsymbol{r}_{i j}^{S V}$ (from frame $C$ ) are measured for all the target points $j=1,2, \ldots, 132$ by triangulation of the detected target pixel locations. Once the corresponding image locations are detected from a stereo pair of images, a direct linear transformation algorithm [39] can be used to carry out the triangulation-determination of a third 3D point.

It is necessary to align the stereo vision measurements ${ }^{C} \boldsymbol{r}_{i j}^{S V}$ to the laser interferometer displacement measurements along the direction of motion of the stage. This is accomplished by estimating the relative orientation (from frame $C$ ) of the stage reference frame $O$, from where the stage displacements are observed by the interferometer. Figure $3 \mathrm{~b}$ ) shows the direction of motion of the stage (unit vector $\widehat{\boldsymbol{u}}_{x}$ ) evaluated from the best-fit lines of the displacements of the target points 
${ }^{C} \boldsymbol{r}_{i j}^{S V}-{ }^{C} \boldsymbol{r}_{1 j}^{S V}$. In addition, planar fits of the displaced horizontal arrays of the target points, shown in Figure 3c), are used to evaluate the upward direction (unit vector $\widehat{\boldsymbol{u}}_{z}$ ) of the stage in frame $C$. By orthogonality of the Cartesian coordinate system, the $y$-direction of the stage is given by $\widehat{\boldsymbol{u}}_{y}=$ $\widehat{\boldsymbol{u}}_{z} \times \widehat{\boldsymbol{u}}_{x}$. Having obtained the stage's directions measured from the stereo vision system, the orientation of the stage reference frame can be expressed as ${ }^{C} \boldsymbol{R}_{O}=\left[\widehat{\boldsymbol{u}}_{x}, \widehat{\boldsymbol{u}}_{y}, \widehat{\boldsymbol{u}}_{z}\right]$. Hence, the stagealigned linear displacements of the target points are given by

$\left[\begin{array}{c}x \\ \delta_{y} \\ \delta_{z}\end{array}\right]_{i j}^{S V}={ }^{C} \boldsymbol{R}_{O}^{-1} \cdot\left[{ }^{C} \boldsymbol{r}_{i j}^{S V}-{ }^{C} \boldsymbol{r}_{1 j}^{S V}\right]$.

The $x$-components of the linear displacements in Equation (2) $x_{i j}^{S V}$ are now comparable with the reference interferometry readings $x_{i}^{L I}$. Therefore, the linear displacement error is obtained from the difference of $x_{i}^{L I}$ and $x_{i j}^{S V}$. In addition to the linear displacement error, Equation (2) gives the straightness errors $\delta_{y}$ and $\delta_{z}$ which represent the respective $y$-axis and $z$-axis deviations of the tracked points from the ideal axis of motion.

The angular displacement error of the stage can also be estimated using the points measured by the stereo vision camera. The orientation ${ }^{C} \boldsymbol{R}_{P}$ of the optical target at any position of the stage can be estimated from the direction vectors of the lines formed by the horizontal and vertical arrays of points on the target plane, shown in Figure 3d). Since the frames $S$ and $P$ are rigidly connected to the moving stage, the relative orientation ${ }^{S} \boldsymbol{R}_{P}$ remains constant and ${ }^{S} \boldsymbol{R}_{P}={ }^{O} \boldsymbol{R}_{P}$ due to the fact that the frame $S$ coincides with $O$ at the first stage position. The value of ${ }^{S} \boldsymbol{R}_{P}$ is evaluated at the first stage position as ${ }^{S} \boldsymbol{R}_{P}={ }^{O} \boldsymbol{R}_{P}={ }^{C} \boldsymbol{R}_{O}^{-1} \cdot{ }^{C} \boldsymbol{R}_{P}$. Finally, the relative change in orientation of the stage with respect to the first stage position can be computed using

${ }^{o} \boldsymbol{R}_{S}={ }^{C} \boldsymbol{R}_{O}^{-1} \cdot{ }^{C} \boldsymbol{R}_{P} \cdot{ }^{S} \boldsymbol{R}_{P}^{-1}$.

From Equation (3), the values of the angular error components can then be evaluated from the elements of the orientation matrix ${ }^{O} \boldsymbol{R}_{S}$ as the extrinsic Euler angles (observed from the fixed frame $O$ shown in Figure 3)[40]

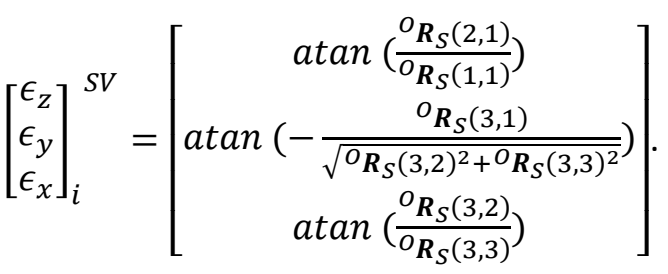

The matrix element ${ }^{o} \boldsymbol{R}_{S}(n, m)$ denotes the $\mathrm{n}^{\text {th }}$ row and $\mathrm{m}^{\text {th }}$ column of a matrix ${ }^{o} \boldsymbol{R}_{S}$, as shown in Equation (4). The three angular error components $\left[\epsilon_{z}, \epsilon_{y}, \epsilon_{x}\right]$ are the yaw, pitch and roll of the stage respectively.

Even though Equations (2) and (4) do not depend on the origin of frame $O$, the origin is necessary for further study on volumetric error in the rest of the section. The origin of $O$ is assigned to the origin of the detected target points at the first stage position, such that the origins of $P$ and $S$ are essentially aligned to the motion axis as the stage moves, with slight deviations investigated as straightness errors. 


\subsection{Discrete volumetric error generation}

Up to this point, transformations have been carried out on the stereo vision measurements to align them with the measurements from the laser interferometer. Here, the homogenous transformation matrix formed by the laser interferometer measurement is used to generate a reference trajectory for the optical target points. The volumetric error associated with the stereo vision is then measured from the reference trajectory. Figure 4a) shows the schema of the laser interferometer trajectory that is applied to all points in frame $O$. The three viewpoints shown in the figure are used to generate the volumetric errors. For each of the three viewpoints, the evaluated difference in positions (error vectors) will need to be transformed to the stereo vision frame from where the volumetric errors are recorded. The equation

$\left[\begin{array}{c}E_{x} \\ E_{y} \\ E_{z} \\ 1\end{array}\right]_{i j}=\left[\begin{array}{cc}{ }^{C} \boldsymbol{R}_{O} & \mathbf{0}_{3 \times 1} \\ \mathbf{0}_{3 \times 1}^{T} & 1\end{array}\right] \cdot\left(\left[\begin{array}{c}x \\ y \\ z \\ 1\end{array}\right]_{i j}^{S V}-\left[\begin{array}{cccc}1 & -\epsilon_{z} & \epsilon_{y} & x \\ \epsilon_{z} & 1 & -\epsilon_{x} & \delta_{y} \\ -\epsilon_{y} & \epsilon_{x} & 1 & \delta_{z} \\ 0 & 0 & 0 & 1\end{array}\right]_{i}^{L I} \cdot\left[\begin{array}{l}x \\ y \\ z \\ 1\end{array}\right]_{1 j}{ }^{S V}\right)$,

gives the volumetric error vector plotted in Figure $4 b$ ), where $\mathbf{0}_{3 \times 1}=[0,0,0]^{\mathrm{T}}$. This displacement error vector is considered to be dependent on the position from the stereo-camera, so that the same error information can be treated as an intrinsic property of the stereo vision system.

a)

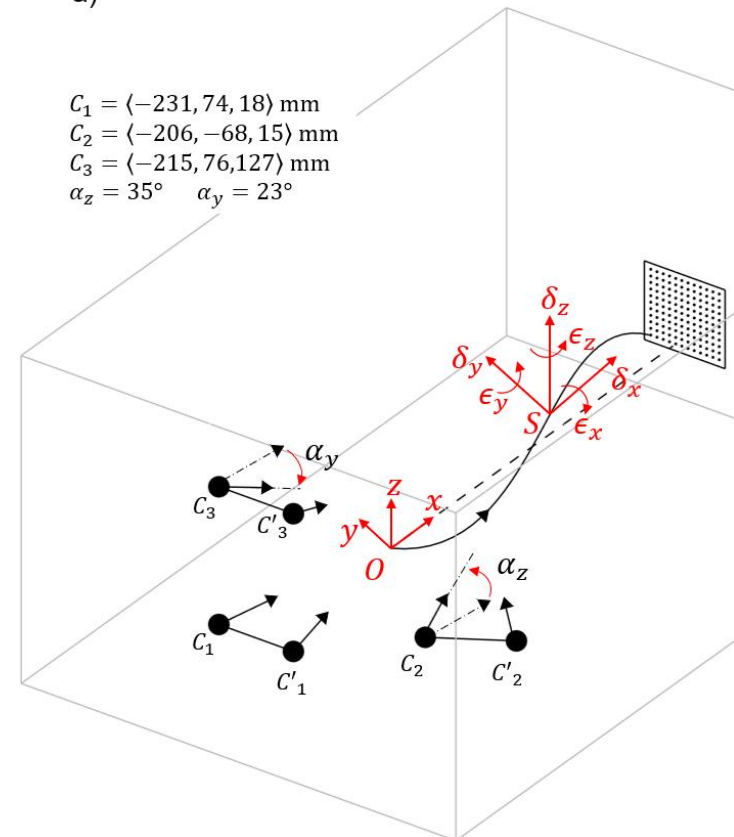

b)

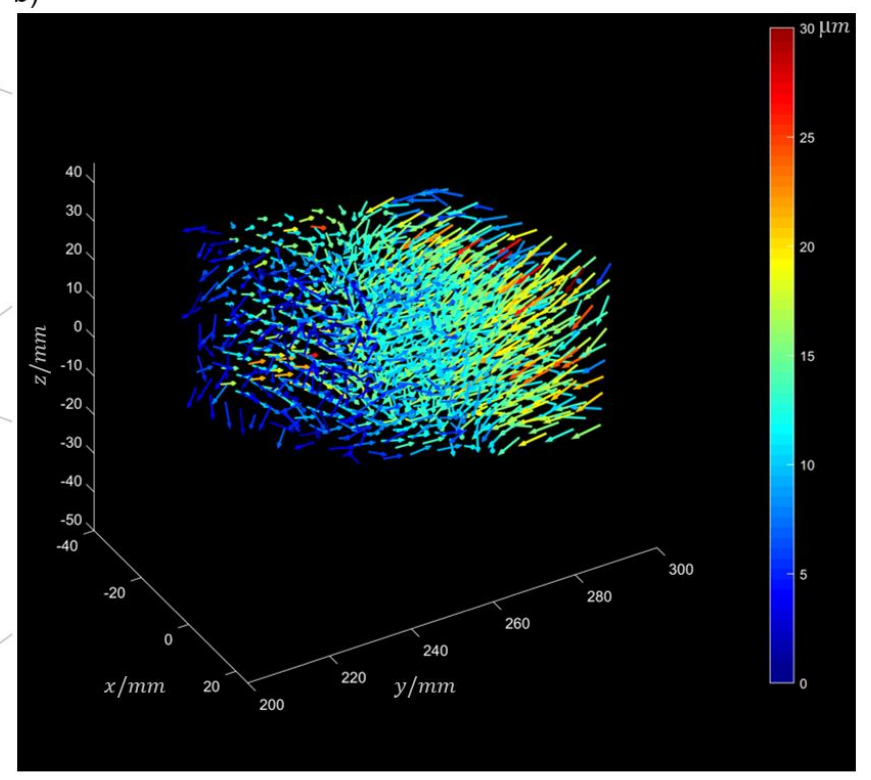

Figure 4. a) Illustration of trajectory of transformed target points observed from three stereo camera positions $C_{1}, C_{2}, C_{3}$ given with respect to $O$. b) Plot of volumetric error vectors in coordinate frame $C_{1}$ target positions. $\alpha_{z}$ and $\alpha_{y}$ are the approximate rotation angle of $C_{2}$ and $C_{3}$ respectively from $C_{1}$, along the respective $z$ and $y$ directions.

The displacement measurement experiment can be repeated with the optical target at different locations on the stage to cover a higher measurement volume. The error vector is discrete and cannot directly provide error information at any desired position; hence, there is need for a continuous model of the error. There is no analytical model for the behaviour of the error vectors, and it is difficult to 
model using elementary mathematical functions [21, 22]. The lack of a spatial relationship with the error components led to investigation of regression and scattered 3D interpolation methods.

\subsection{Regression modelling of volumetric error}

Most research on volumetric error is targeted at machine tools that operate in Cartesian coordinates, where error can be traced to individual axes $[17,21]$. Measured error components in the homogenous transformation matrices of these systems are easily interpolated to generate complete volumetric error. However, stereo vision systems operate by synthesising central projections of a 3D scene to a 2D image plane [39], making it difficult to implement a homogeneous transformation model as there are no physically moving components that connect a triangulated point in a scene with an observed stereo image points. The volumetric error computed from Equation (5) is experimentally obtained and relates to spatial positions in an unknown, noisy and non-linear pattern that is difficult to model analytically.

Artificial neural networks can be used to model complex and non-linear behaviour in the presence of noisy data [41]. The performance of neural network is compared with interpolation methods later in this section. While interpolation of the discrete errors at a desired location can be used, neural network is shown to be better suited for regression of the error. Hence, a multi-input multi-output feedforward neural network has been chosen to model the generated volumetric error. After testing several networks with different combinations of number of hidden layers and number of neurons, the shallow neural network shown in Figure 5 was chosen. The neural network has three inputs (the components of the position vector from the stereo-camera), fifteen hidden layer neurons and three output layer neurons used to compute the three components of the volumetric error.

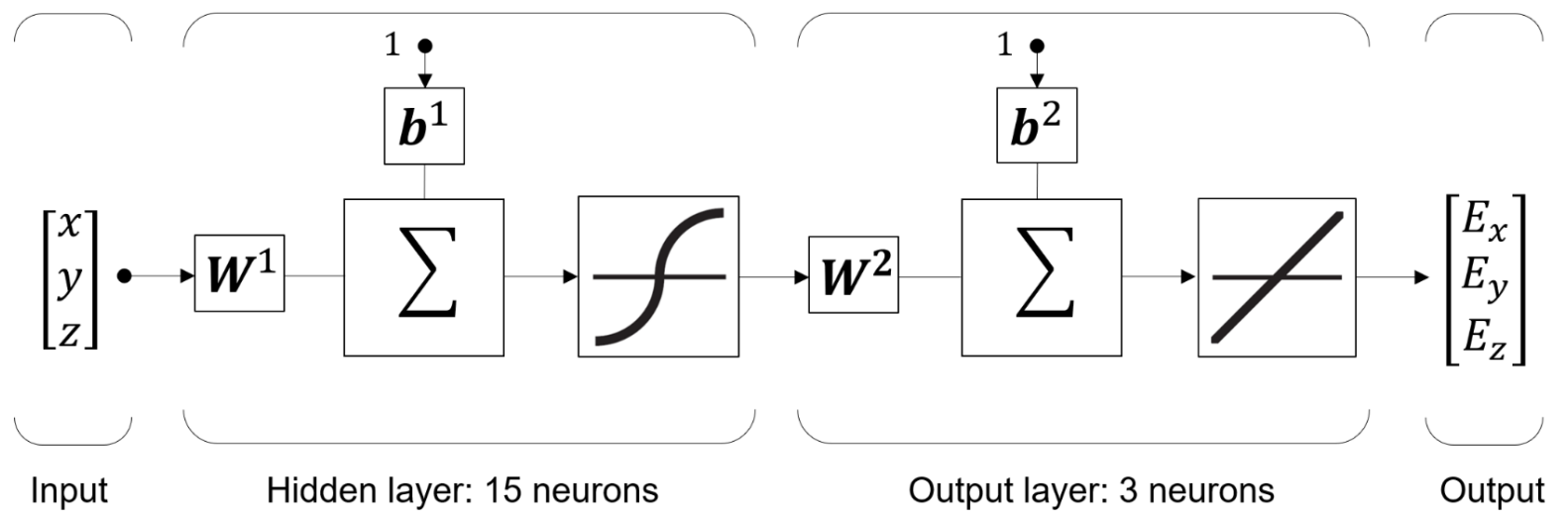

Figure 5. Neural network showing input position coordinate values, a hidden layer with fifteen neurons and an output layer with three neurons to generate the volumetric error as output. $\boldsymbol{W}^{1}$ and $\boldsymbol{b}^{1}$ are the hidden layer weight matrix and bias vector respectively. $\boldsymbol{W}^{2}$ and $\boldsymbol{b}^{2}$ are the output layer weight matrix and bias vector respectively.

The hidden layer of the neural network has a hyperbolic sigmoid activation function and the output layer has a linear function. The hyperbolic sigmoid function maps weights to a zero-centred higher resolution range (-1 to 1 ) compared to the popular exponential sigmoid function which maps to a 0 to 1 range. A batch gradient descent learning algorithm with Bayesian regularisation [41] was used to train the weights and biases of the neural network in Figure $5.70 \%$ of the position and error data obtained from of the five experimental campaigns repeated at each of the three positions shown in Figure 4a were randomly selected to train the neural network. This training data is, to some extent, representative of the repeatability and scene-dependency of the stereo vision system. The 
performance of the trained neural network was tested on the remaining validation data; the mean squared error of training and validation data are shown in Figure 6a). The distribution of the error of prediction $\left(\Delta E_{x}, \Delta E_{y}, \Delta E_{z}\right)$ on the validation data is given in Figure $\left.6 \mathrm{~b}\right)$, along with the standard deviations of the error of predictions. These validation error deviations are $2.7 \mu \mathrm{m}, 4.0 \mu \mathrm{m}$ and $1.7 \mu \mathrm{m}$ along $x, y$ and $z$ repectively. As expected from Monte-Carlo simulations $[1,11]$, the uncertainties along the principal axes of the cameras ( $y$-axis for the left camera) are higher and are, therefore, predicted with higher errors. It is important for the majority of the predicted volumetric errors to have error magnitudes that are comparable to the random error of the stereo vision measurements. An empirical estimation of randomness can be made from the repeatability of the measurements discussed in Section 5.

The reason for choosing neural network regression over interpolation methods is that interpolation methods like Delaunay triangulation and natural neighbour [42] predict the errors with higher validation errors, manifested by higher standard deviations of over $30 \%$ for each error component. Interpolation by natural neighbour method of the same training data at the validation positions shows standard deviation of validation errors of $3.6 \mu \mathrm{m}, 5.4 \mu \mathrm{m}, 2.3 \mu \mathrm{m}$ along $x, y$ and $z$ respectively. In addition, significant instability is observed when the interpolation and validation data are resampled.

a)

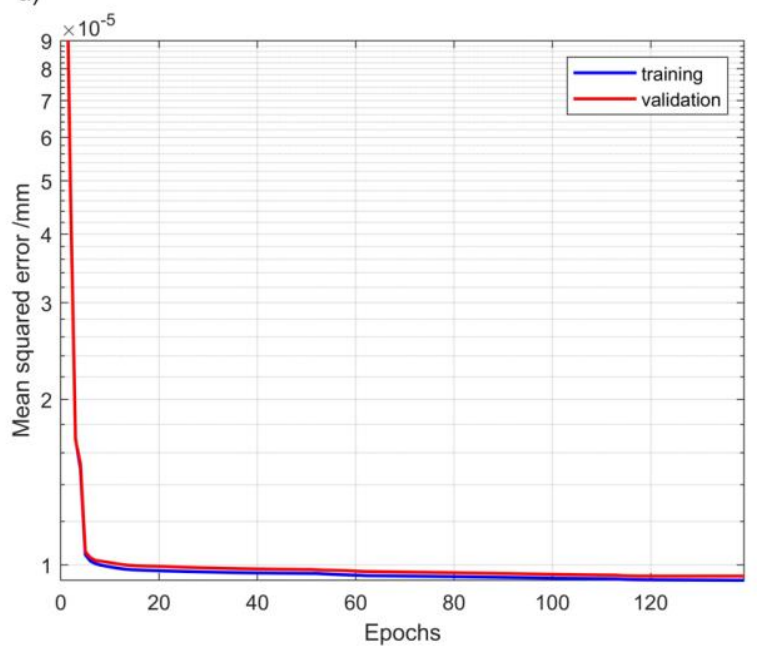

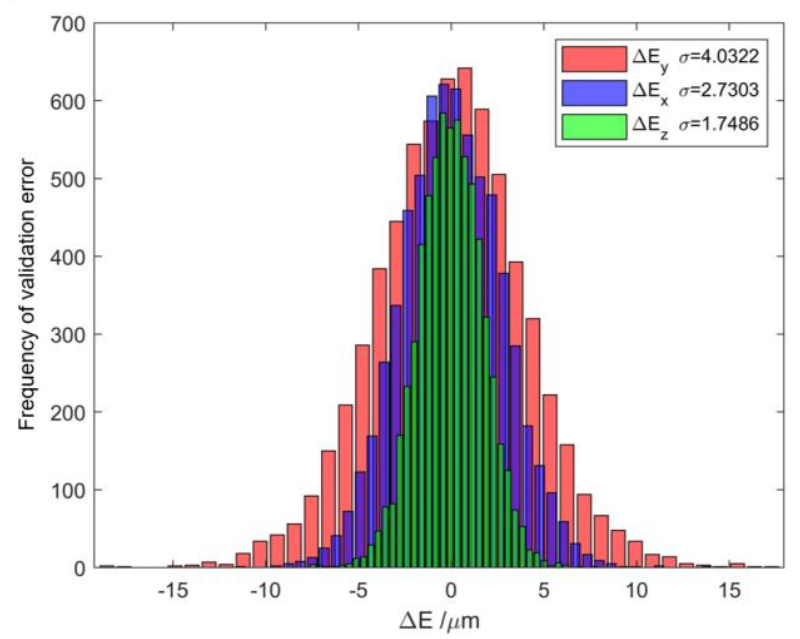

Figure 6. a) Performance of the implemented neural network given as mean squared error of training and validation given against epochs (learning cycles of iterative update of trained weights and biases). b) Distribution of the validation error, which represents the difference between predicted volumetric errors and the actual errors. The legend shows the standard deviation of the components of the validation errors $\left(\Delta E_{x}, \Delta E_{y}, \Delta E_{z}\right)$.

\section{Error correction applied to photogrammetry}

Referring to Figure 2a), the artefact rotates in a clockwise direction relative to the fixed cameras; an equivalent artefact-centric perspective where the cameras rotate by equal angles in the opposite anticlockwise direction is illustrated in Figure 7a). Since photogrammetry reconstructs objects from varying imaging viewpoints, the artefact-centric perspective is adopted. Images of the artefact captured at $6^{\circ}$ rotational intervals are fed into the OpenMVG SfM pipeline. Image features are detected using a local image descriptor, known as the scale invariant feature transform (SIFT) [43], and matched using 
cascade hashing [44]. The orientation and position of the rotary stage, relative to the stereo-camera, were predetermined through a geometric characterisation process. The characterisation of the rotary stage was carried out by tracking markers rotated on the stage and analysing their trajectory deviations from ideal circular paths. The rotary axis was found to be characterised by run-out of less than $8 \mu \mathrm{m}$ and axial wobble of less than $0.01^{\circ}$. Knowing the rotation axis of the rotary stage and the angles of rotation makes it possible to compute the artefact-centric poses of the imaging viewpoints accurately. In addition, measurement of the artefact-centric poses enables computation of the baseline distances among imaging viewpoints, which is essential in the selection of image pairs for accurate feature extraction and matching. In photogrammetry, there is usually a compromise between accuracy and object visibility; while large baselines result in detection of features with higher accuracy, smaller ones ensure more common features are visible $[11,45,46]$. Figure $7 b$ ) outlines how several processes are integrated with the SfM pipeline. By employing the matched features and imaging poses, triangulation is carried out using an OpenMVG subroutine to compute 3D points.

a)

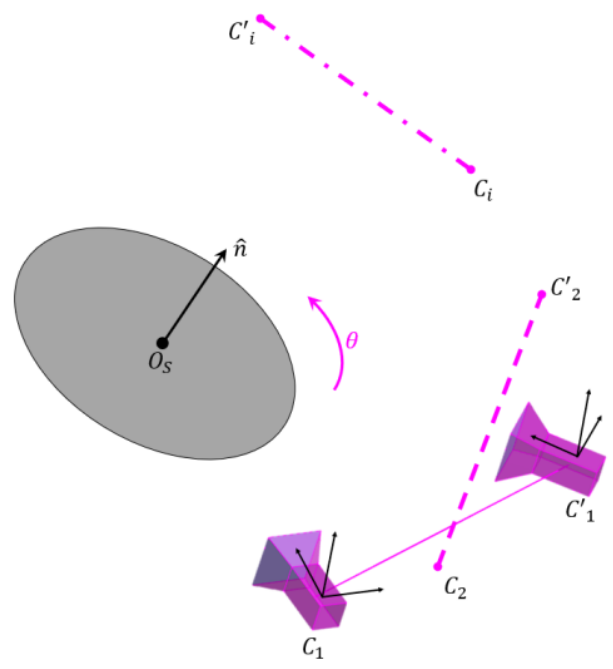

b)

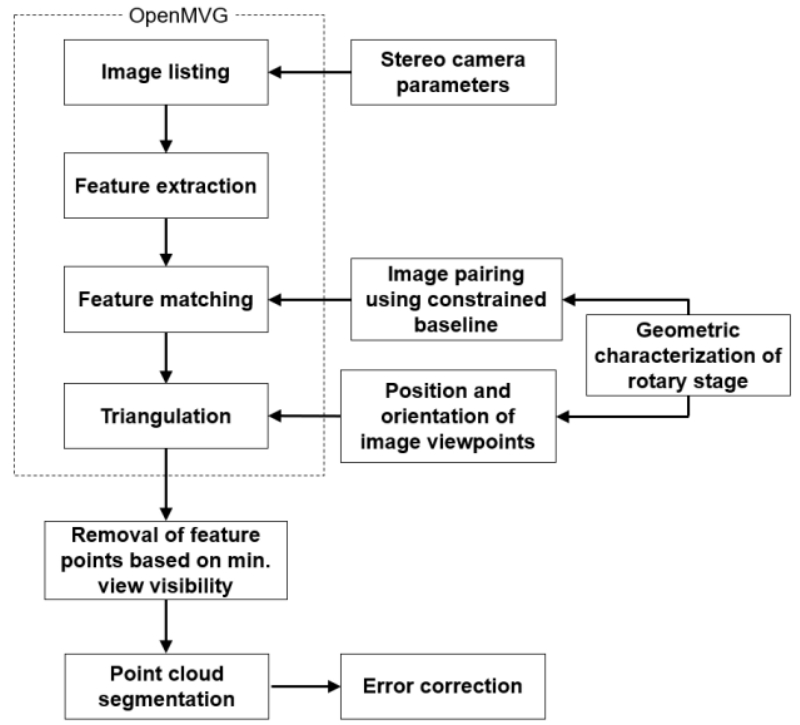

Figure 7. a) The artefact-centric rotation of imaging viewpoints. b) Flowchart summary of the SfM pipeline.

Besides control of the image viewpoints and baseline distances, false feature matches are mitigated by removing features that appear in fewer than a number of viewpoints (number of viewpoints $\geq 3$ ). The higher the number of viewpoints that agree on a feature, the lower the probability of a false positive match of the feature. 


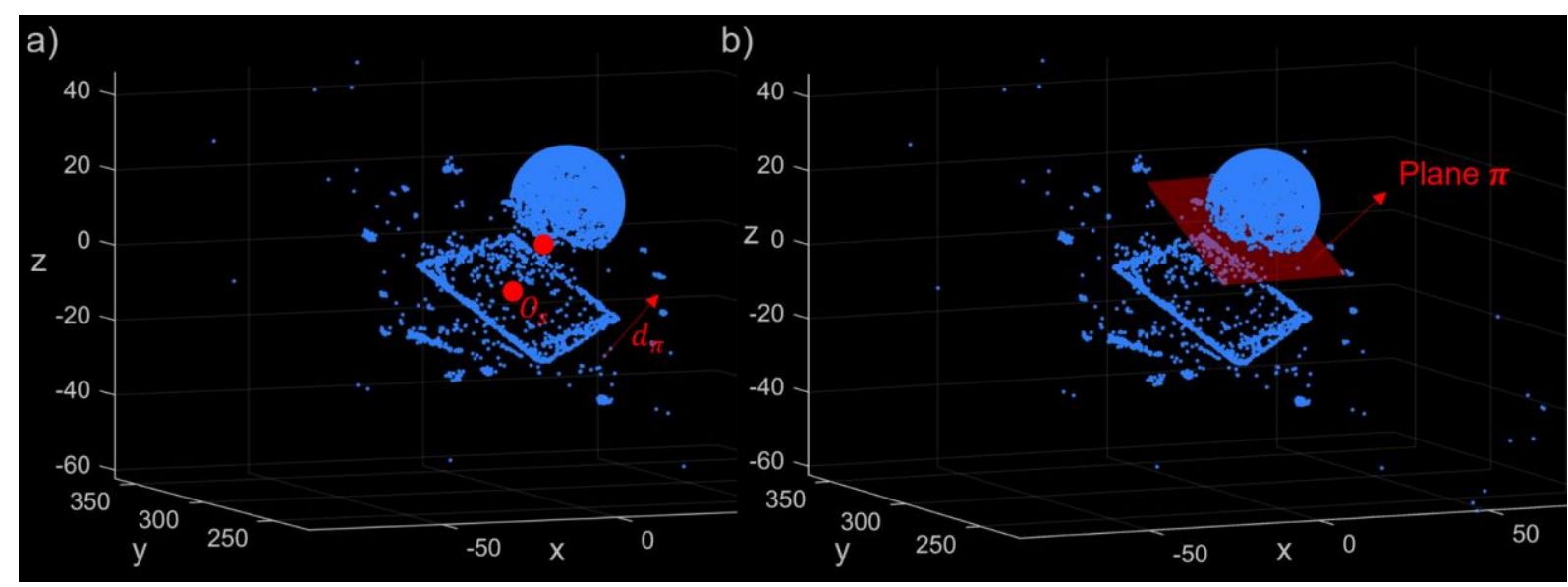

Figure 8. Point cloud segmentation process, where a) a point on a plane is found, b) segmentation plane is constructed.

Not all the reconstructed points are expected to be on the spherical artefact hence, an automated point cloud segmentation process, shown in Figure 8, was developed. A point, with position vector $\boldsymbol{r}_{\pi}$, on a desired segmentation plane is created along the stage rotation axis $(\widehat{\boldsymbol{n}})$ with a distance $d_{\pi}=22 \mathrm{~mm}$ from the stage centre point $O_{s}, \boldsymbol{r}_{\pi}=\boldsymbol{r}_{O_{s}}+d_{\pi} \widehat{\boldsymbol{n}}$. The center-position vector and the unit rotation direction vector of the stage are denoted by $\boldsymbol{r}_{O_{S}}$ and $\widehat{\boldsymbol{n}}$ respectively. The segmentation plane $\boldsymbol{\pi}$ is chosen to have normal $\widehat{\boldsymbol{n}}$ and can be expressed in the homogenous four-vector representation [39] as: $\boldsymbol{\pi}=\left[\widehat{\boldsymbol{n}}^{T} \mid-\boldsymbol{r}_{\pi}^{T} \cdot \widehat{\boldsymbol{n}}\right]^{T}$. The decision on whether a point, represented by position vector $\boldsymbol{r}_{p}$, is on the spherical region is made from the condition $\boldsymbol{r}_{p}^{T} \cdot \widehat{\boldsymbol{n}}>\boldsymbol{r}_{\pi}^{T} \cdot \widehat{\boldsymbol{n}}$.

Finally, the trained neural network regression model $\boldsymbol{E}^{N N}$ is used to correct the error of each 3D point. For a 3D feature that appears in an image view at rotation angle $\theta$, the corrected 3D position of the feature point is given by

$\boldsymbol{r}_{p}^{\text {corr }}=\boldsymbol{R}(\widehat{\boldsymbol{n}}, \theta) \cdot\left[\boldsymbol{r}_{p}^{\prime}-\gamma \boldsymbol{E}^{N N}\left(\boldsymbol{r}_{p}^{\prime}+\boldsymbol{r}_{O_{s}}\right)\right]+\boldsymbol{r}_{O_{s}}$.

Equation (6) adjusts the transformed 3D point, $\boldsymbol{r}_{p}^{\prime}=\boldsymbol{R}(\widehat{\boldsymbol{n}}, \theta)^{-\mathbf{1}} \cdot\left(\boldsymbol{r}_{p}-\boldsymbol{r}_{O_{s}}\right)$, to the original instance where the image was acquired and subtracts the predicted error from the transformed 3D position. A correction factor $\gamma$ is used to scale the correction and the corrected point is transformed back to the world coordinate frame. The transformations are carried out using the rotation matrix $\boldsymbol{R}(\widehat{\boldsymbol{n}}, \theta)$ that is evaluated from the Euler-Rodrigues formulation [47] using $\widehat{\boldsymbol{n}}$ and $\theta$. The correction is carried out using the direct prediction values for which $\gamma=1$ and for a least-squares optimisation of the correction factor given by

$\min _{\gamma}\left(\left\|\boldsymbol{r}_{p}^{\text {corr }}-\boldsymbol{r}_{s p h}\right\|-\rho_{s p h}\right)$

where $\boldsymbol{r}_{s p h}$ is the centre-position of a fitting sphere with radius $\rho_{s p h}$. The value of the optimised correction factor from Equation (7) is found to be $\gamma=1.9085$. 


\section{Results}

The results of the stereo vision tracking error, photogrammetric application and error correction are discussed in this section.

\subsection{Analysis of stage displacement measurements}

The linear and angular displacements of the linear stage, evaluated from Equations (2) and (4) respectively, are assessed against the linear interferometer measurements. For verification purposes, the displacement experiment was repeated five times. Hence, both the accuracy and repeatability of the stereo vision system in measuring the linear displacement of the stage are analysed. Images containing the optical targets were captured while the laser interferometer readings were recorded at ten different positions of the stage.

Figure 9 shows the linear displacement error and the estimation of straightness error, with the maximum and minimum values of the repeated measurements shown as error bars. The results are evaluated in the stereo-camera position $C_{1}$ given in Figure $4 a$ ). The linear error in Figure 9a) is less than $15.1 \mu \mathrm{m}$ and varies by a maximum value of $5.2 \mu \mathrm{m}$. When the regression model is applied to the stereo vision measurement to correct errors, systematic error is shown to diminish in Figure 9b). The straightness error in $z$-direction is a little higher (by approximately $3 \mu \mathrm{m}$ ) than that in $y$-direction. While the $z$ straightness error may be affected by Abbe offset error $(<2 \mu \mathrm{m})$, it is feasible for the stage to have non-uniform straightness in the two directions.
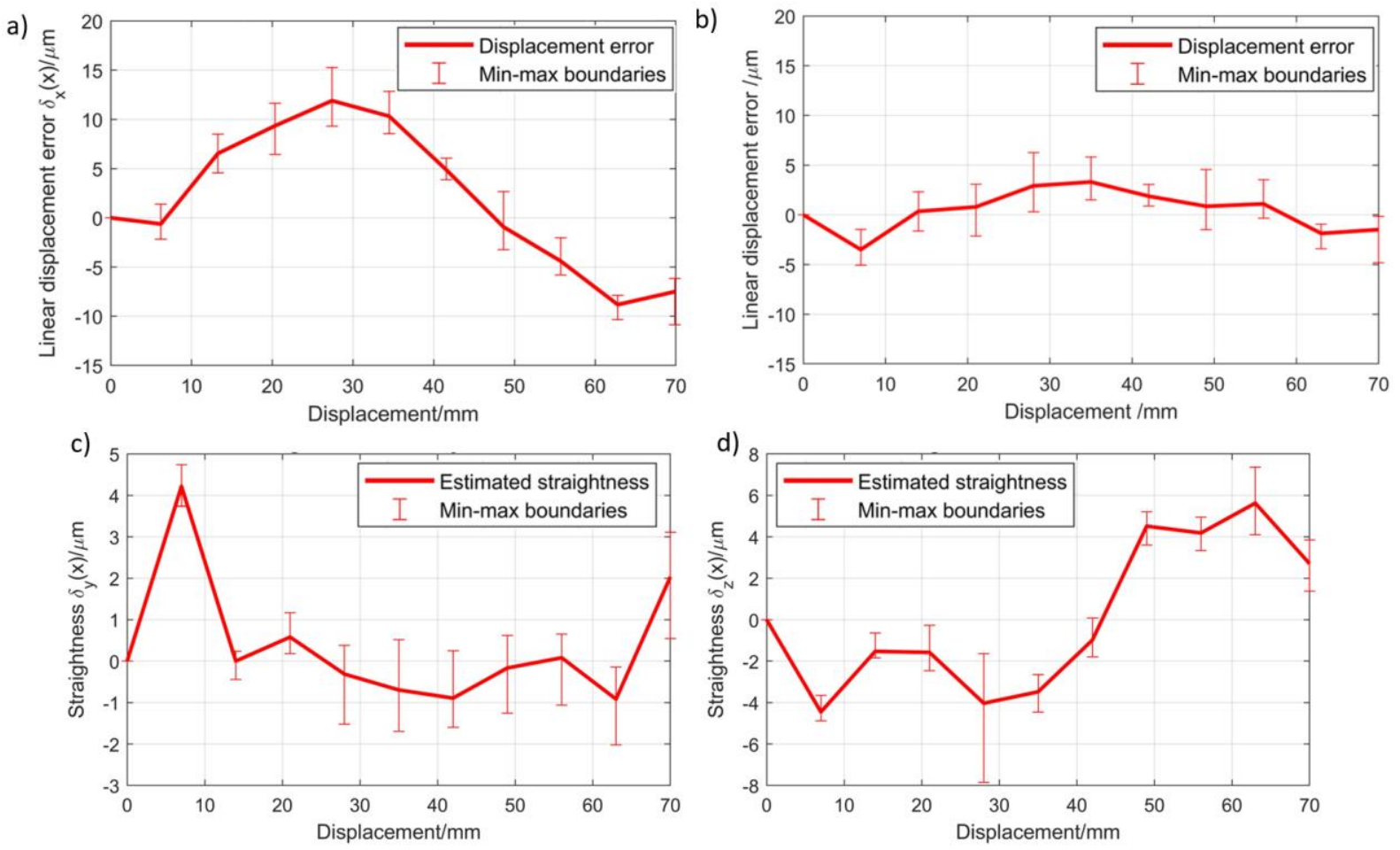

Figure 9. a) Measured stereo vision displacement error in linear direction $x$-direction. Correction of stereo vision measurements using regression model is shown to reduce systematic error significantly in (b). Measured stereo vision straightness error in c) $y$-direction and d) $z$-direction of the linear stage. 
Figure 10 shows the mean laser interferometer measurements (in solid red lines) with error bars showing the extreme readings of the angular measurements. The corresponding angular displacements computed from the stereo vision system are also shown. Variations in the displacement measurements of the stereo vision system are indicated by the envelope plots, which indicate the minimum and maximum results across the five experiments. The measurement results in Figure 10 were obtained from the same stereo-camera position, however, the following observations are also valid for the other stereo vision positions [48]. A small angular displacement of less than $100 \mu \mathrm{rad}$ in yaw and pitch was observed by the interferometer. The yaw and pitch angles of the stereo vision measurement in Figure 10a) and b) were significantly overestimated when compared to the laser interferometer readings. The estimated roll values in Figure 10c) remain unverified because the angular setup of the laser interferometer could not be used for roll measurements. The spread of the stereo vision measurements is, on the whole, up to five times wider than the spread in the laser interferometer results.
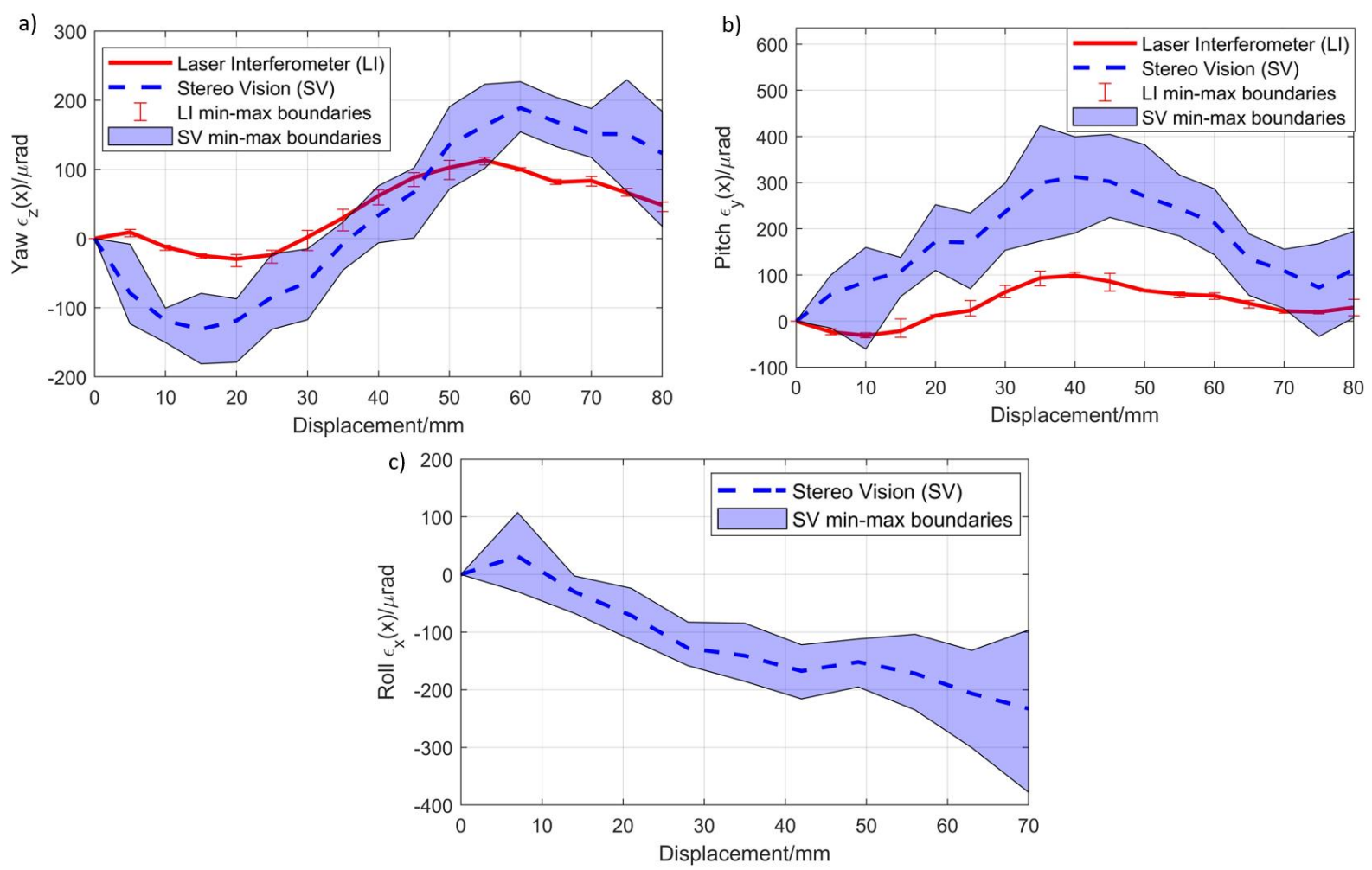

Figure 10. Angular measurement results showing error in (a) yaw, (b) pitch and (c) roll in configuration A measured by laser interferometer (LI) and stereo vision (SV).

The pattern of the displacement error persists when the experiment is carried out at various positions of the stereo vision system [48], indicating the existence of intrinsic systematic behaviour. The linear displacement error (along $x$ ) is more dominant and significant than the straightness and angular errors. While the linear displacement error remains less than $20 \mu \mathrm{m}$, the repeatability is below $6 \mu \mathrm{m}$. The magnitude of the systematic error is reduced by over up to $50 \%$ in Figure $9 \mathrm{~b}$ ) after error correction. As seen in the figure, the systematic error becomes overshowed by the repeatability of the stereo vision system; therefore, further error-correctional endeavours become insignificant. 


\subsection{Photogrammetry results}

The parameters that significantly affect the photogrammetric application of the stereo vision system are the length of imaging baseline pairs and number of agreeing feature viewpoints. This section studies the reconstruction results across a range of these parameters as well as the effects of the error correction. The quality of measurement of the 3D points is reported in accordance with the VDI/VDE 2634 definition of probing form and size errors [49].

Figure 11a) shows the total number of points on a sphere using four ranges of baseline distances used for image feature matching. The number of points reduces significantly when the minimum number of feature views is increased from three. The rate of decrease of the number of points increases with higher baseline lengths. For all the configurations analysed, the number of points must be greater than 2500 to ensure enough coverage of the spherical surface.
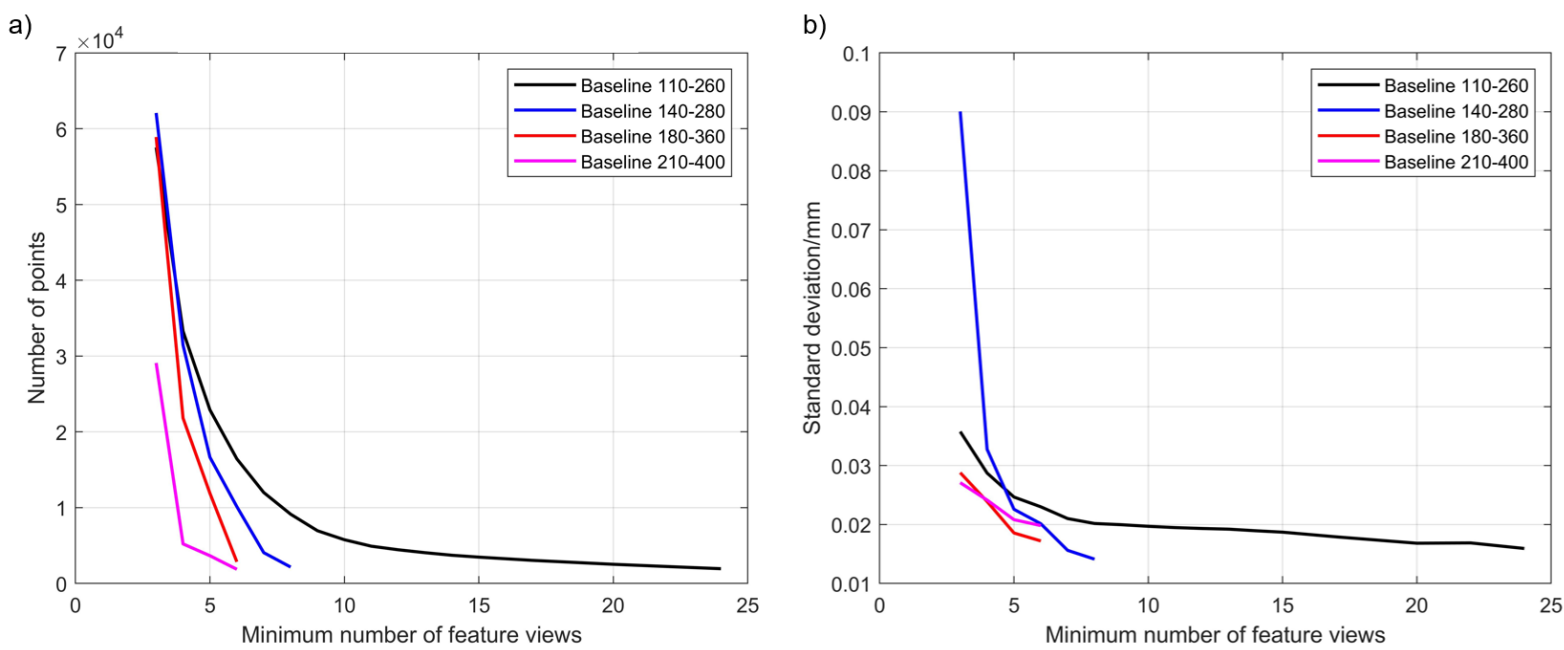

Figure 11. Variations in, a) number of point and b) standard deviation of radial distance from fitting sphere, for various limit on the number of feature appearances in imaging viewpoints using four ranges of baseline distance.

Figure 11b) shows the standard deviation of radial distances of points from a fitted sphere. While the decrease in the number of points is undesireable, there is a benefit-a decrease in outliers and mismatches-in choosing points with a higher number of viewpoint detections. Hence, more accurate 3D points (resulting in lower form error) are obtained at the cost of the number of the points. The configurations with baselines between $140 \mathrm{~mm}$ to $280 \mathrm{~mm}$ and $180 \mathrm{~mm}$ to $360 \mathrm{~mm}$ show more improvement in standard deviation while maintaining a sufficient number of points (>2800). 


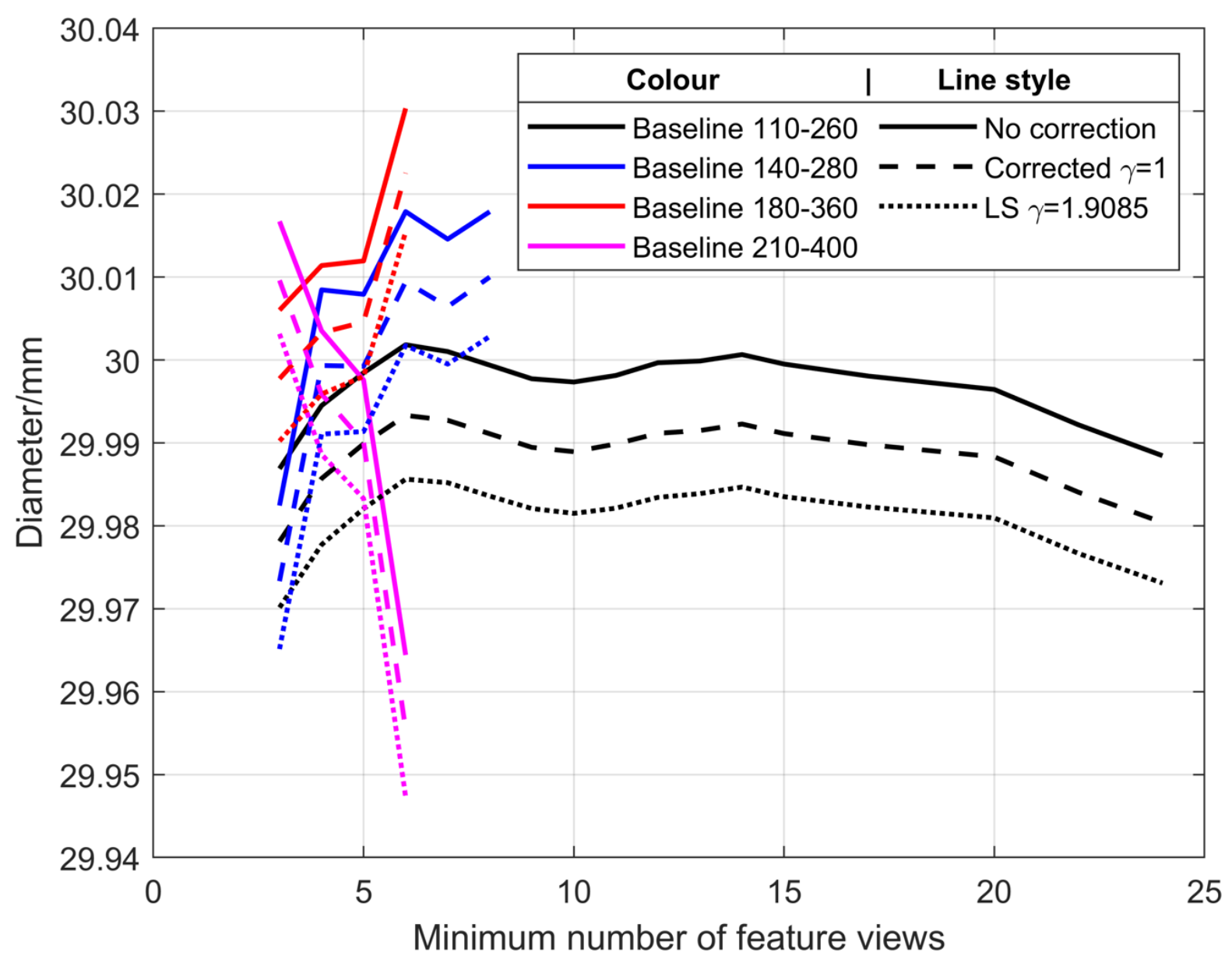

Figure 12. Measurement diameter of fitting sphere for different ranges of baseline lengths using no error correction, error correction employing predictions $(\gamma=1)$, and error correction with leastsquares-optimized factor $\gamma=1.9085$.

The variations of the fitted spherical diameter obtained from the reconstructed points are shown in Figure 12. Relative to the calibrated diameter of $29.952 \mathrm{~mm}$ (with radial $\pm 0.032 \mathrm{~mm}$ ), an improved diameter measurement over the various photogrammetry configurations is observed. The effect of using the least-squares optimised correction factor of $\gamma=1.9085$ is that it increases the effect of the predicted correction. 


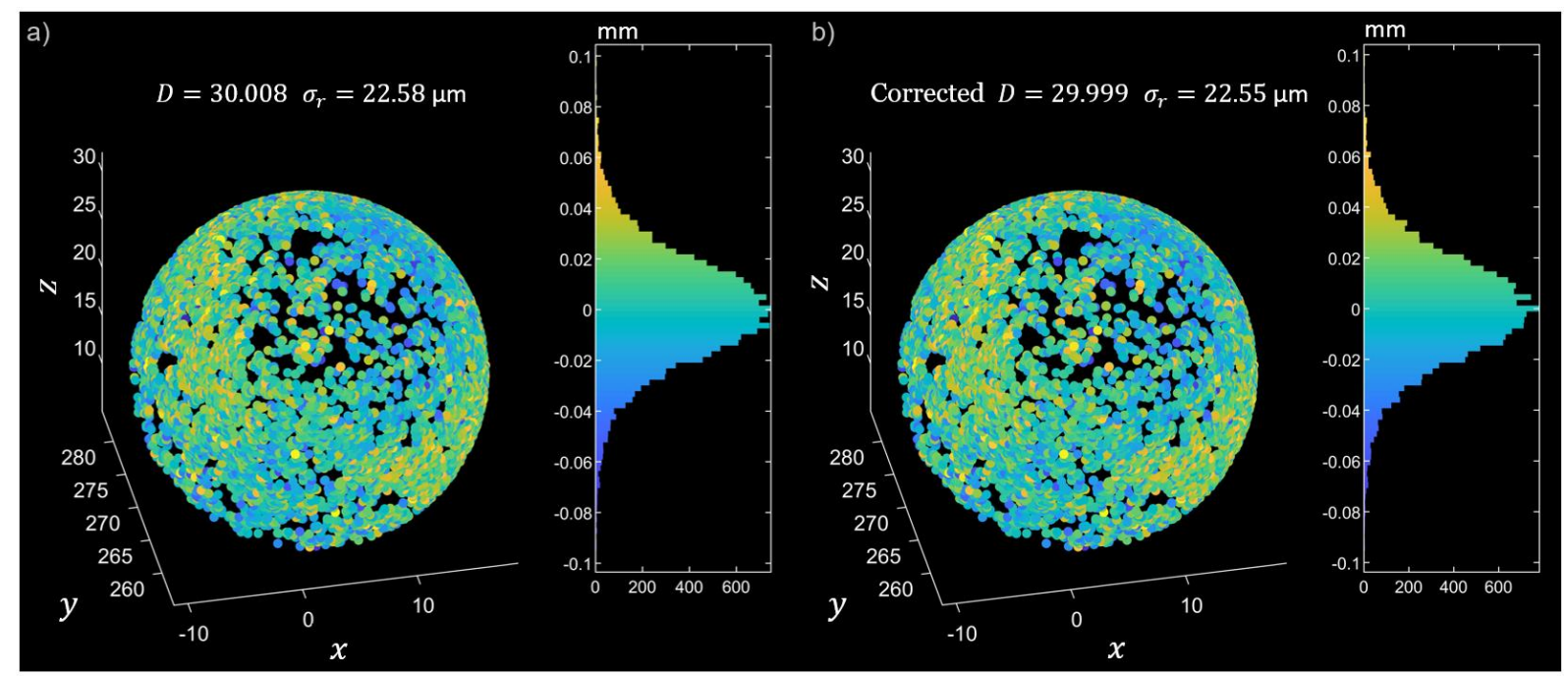

Figure 13. Distribution of radial deviation of points from a sphere for baselines distances between $140 \mathrm{~mm}$ to $280 \mathrm{~mm}$ with features existing in more than five viewpoints. $\sigma_{r}$ is the radial standard deviation of radial distances from the fitting sphere.

The applied correction does not only correct the diametric size of the measured points but also improves how well the points fit to a sphere which, can be seen from the small improvement in radial standard deviation of the deviation of points from the fitting sphere (Figures 13 and 14). Figure 13 shows the distribution of the radial deviation of the points, with and without error correction for baselines between $140 \mathrm{~mm}$ to $280 \mathrm{~mm}$, set at minimum feature views of five. Similar improvement in radial deviation can be seen when the number of minimum views is increased to seven (Figure 14). The distribution of radial deviation in Figure 14b) is within $\pm 60 \mu \mathrm{m}$, which, compared to the CMM measurement $( \pm 32 \mu \mathrm{m})$, is roughly two times larger. However, over $95 \%$ of the points are within the CMM radial deviation range.

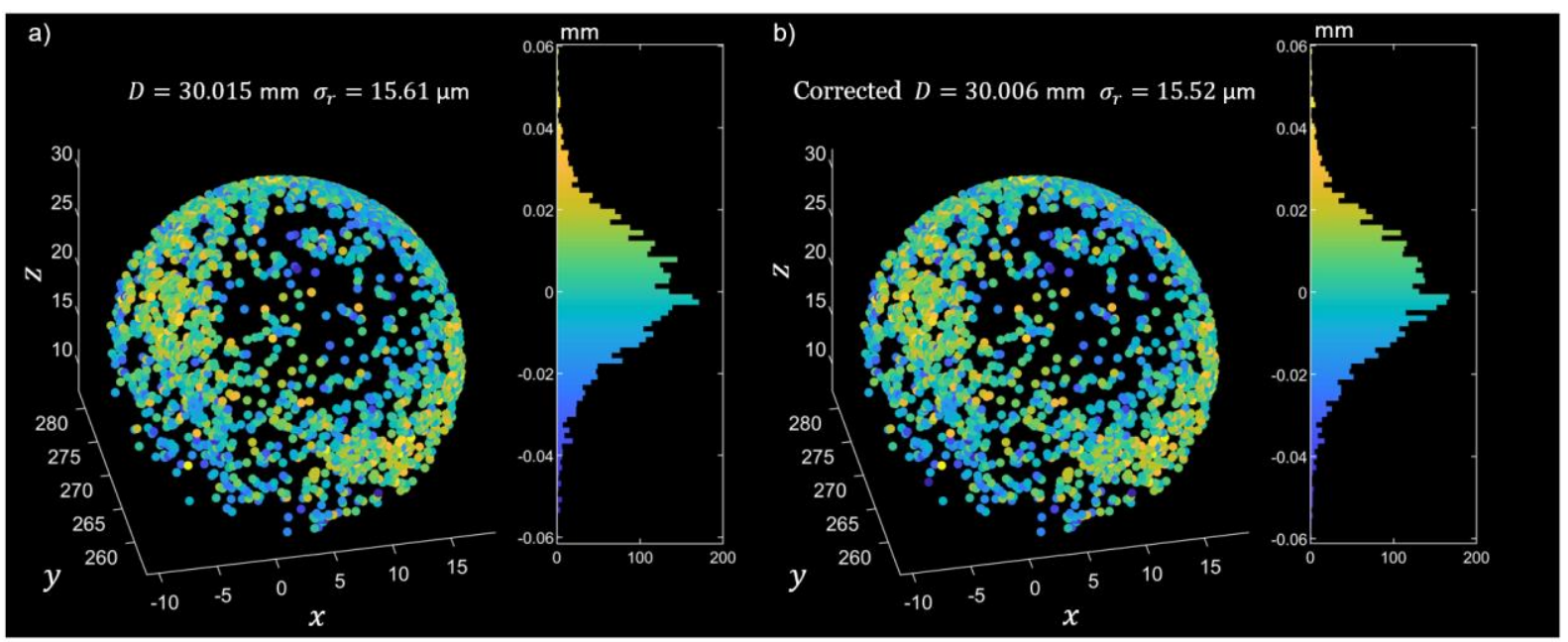

Figure 14. Distribution of radial deviation of points from a sphere for baselines distances between $140 \mathrm{~mm}$ to $280 \mathrm{~mm}$ with features existing in more than seven viewpoints. $\sigma_{r}$ is the radial standard deviation of radial distances from the fitting sphere. 
For some chosen configuration parameters of the photogrammetric measurement given in Table 1, the VDI/VDE 2634 probing form and size quality are evaluated. The form error is defined as the range of radial distances of the measured points from a fitting sphere and the size error is defined as the difference between the measured diameter and a calibrated diameter. The size of error reduction from the no-correction case $(\gamma=0)$, given in square brackets, are up to $30 \%$ for size error and up to $0.6 \%$ for form error.

Table 1. Summary of photogrammetry 3D measurement results showing reduction in error (shown in square brackets) for error correction using various configuration parameters.

\begin{tabular}{|c|c|c|c|c|c|c|c|}
\hline \multirow{4}{*}{ Parameters } & & $\mathrm{C} 1$ & $\mathrm{C} 2$ & $\mathrm{C} 3$ & $\mathrm{C} 4$ & $\mathrm{C} 5$ & $\mathrm{C} 6$ \\
\hline & Baseline/mm & $110-260$ & $140-280$ & $140-280$ & $140-280$ & $180-360$ & $210-400$ \\
\hline & $\begin{array}{l}\text { Minimum feature } \\
\text { views }\end{array}$ & 14 & 5 & 5 & 7 & 6 & 5 \\
\hline & Correction factor $\gamma$ & 1 & 1 & 1.9085 & 1 & 1 & 1 \\
\hline \multirow{5}{*}{ Results } & Diameter/mm & 29.992 & 29.999 & 29.991 & 30.006 & 30.023 & 29.990 \\
\hline & Number of points & 3718 & 16645 & 16645 & 4064 & 2858 & 3656 \\
\hline & $\begin{array}{l}\text { Size error } / \mu \mathrm{m} \\
\text { [reduction } / \% \text { ] }\end{array}$ & $\begin{array}{c}40.3 \\
{[17.2]}\end{array}$ & $\begin{array}{c}47.2 \\
{[15.5]}\end{array}$ & $\begin{array}{c}39.4 \\
{[29.6]}\end{array}$ & $\begin{array}{c}54.4 \\
{[13.0]}\end{array}$ & $\begin{array}{c}70.6 \\
{[9.95]}\end{array}$ & $\begin{array}{c}38.1 \\
{[16.4]}\end{array}$ \\
\hline & $\begin{array}{l}\text { Form error/ } / \mu \mathrm{m} \\
\text { [reduction } / \% \text { ] }\end{array}$ & $\begin{array}{l}266.9 \\
{[0.42]}\end{array}$ & $\begin{array}{l}204.5 \\
{[0.10]}\end{array}$ & $\begin{array}{l}204.3 \\
{[0.18]}\end{array}$ & $\begin{array}{l}119.4 \\
{[0.38]}\end{array}$ & $\begin{array}{l}109.1 \\
{[0.62]}\end{array}$ & $\begin{array}{l}205.8 \\
{[0.46]}\end{array}$ \\
\hline & $\begin{array}{l}\text { Form std. dev. / } \mu \mathrm{m} \\
\text { [reduction } / \% \text { ] }\end{array}$ & $\begin{array}{l}18.94 \\
{[0.02]}\end{array}$ & $\begin{array}{l}22.55 \\
{[0.12]}\end{array}$ & $\begin{array}{l}22.53 \\
{[0.20]}\end{array}$ & $\begin{array}{l}15.52 \\
{[0.58]}\end{array}$ & $\begin{array}{l}17.15 \\
{[0.37]}\end{array}$ & $\begin{array}{l}20.77 \\
{[0.25]} \\
\end{array}$ \\
\hline
\end{tabular}

The configuration $\mathrm{C} 1$, with small baseline setting and high number of viewpoint agreements, has the worst form error due to the higher uncertainty of measured 3D points. A reduced size error is observed in the $\mathrm{C} 3$ configuration when the correction factor in $\mathrm{C} 2$ is increased. Enforcing the minimum number of feature viewpoints from five to seven in C4 diminished the form error to approximately $120 \mu \mathrm{m}$ at the cost of decreased number of points. A similar size of form error is obtained using the higher range of baselines in $\mathrm{C} 5$. The highest set of baselines in $\mathrm{C} 6$ results in higher form error compared to the sets of baselines in all of the configurations $\mathrm{C} 2$ to $\mathrm{C} 5$.

\section{Conclusions}

This paper presents a stereo vision error correction scheme from laser interferometer calibration of linear and angular displacements tracked from a linear stage. First, the error of the stereo vision system in displacement measurement is shown to manifest systematic trends. Consequently, the volumetric error of the stereo vision system is generated, and a regression-type neural network is trained to model the error. The volumetric error model is then implemented in error correction of the photogrammetric 3D measurement of a spherical artefact. The performance of the error correction is reflected in the reduced size and form error of the reconstructed artefact's point cloud. Around $10 \%$ to $30 \%$ reduction in magnitude of the error was observed while over $95 \%$ of the measured points remained within the calibrated form error bounds. 


\section{Acknowledgements}

The authors would like to thank EPSRC (Grants EP/ M008983/1 and EP/L016567/1) and the Higher Education Innovation Fund (HEIF) through a Hermes Fellowship for funding this work.

\section{References}

[1] Sims-Waterhouse D, Isa MA, Piano S, Leach RK. Uncertainty model for a traceable stereophotogrammetry system. Precis. Eng. 2019.

[2] Luhmann T, Robson S, Kyle S, Harley I. Close Range Photogrammetry: Principles, Techniques and Applications. Whittles Publishing; 2011.

[3] Stephen S, Nick P. Passive 3D imaging. In: Nick P, Yonghuai L, Peter B. 3D Imaging Analysis and Applications, Springer; 2012;36-95.

[4] Stavroulakis PI, Leach RK. Review of post-process optical form metrology for industrial-grade metal additive manufactured parts. Rev. Sci. Instrum. 2016;87:041101.

[5] Möller C, Christian H, Hasmukhbhai N. Enhanced absolute accuracy of an industrial milling robot using stereo camera system. Procedia Technol. 2016;26:389-398.

[6] Luhmann T. Close range photogrammetry for industrial applications. ISPRS J. Photogramm. Remote Sens. 2010;65:558-569.

[7] Sankowski W, Włodarczyk M, Kacperski D, Grabowski K. Estimation of measurement uncertainty in stereo vision system. Image Vis. Comput. 2017;61:70-81.

[8] Bräuer-Burchardt C, Möller M, Munkelt C, Heinze M, Kühmstedt P, Notni G. On the accuracy of point correspondence methods in three-dimensional measurement systems using fringe projection. Opt. Eng. 2013;52:063601.

[9] Fooladgar F, Samavi S, Soroushmehr SMR, Shirani S. Geometrical analysis of localization error in stereo vision systems. IEEE Sens. J. 2013;13:4236-4246.

[10] Luhmann T. 3D Imaging - How to achieve highest accuracy. Proc. SPIE 2011;8085:1-11.

[11] Di Leo G, Liguori C, Paolillo A. Propagation of uncertainty through stereo triangulation. 2010 IEEE Int. Instrum. Meas. Technol. Conf. Proc. 2010:12-17.

[12] Isa MA, Lazoglu I. Design and analysis of a 3D laser scanner. Measurement 2017;111:122-133.

[13] Chiodini S, Marco P, Giubilato R, Salviolli F, Berrera M, Franceschetti P, et al. Camera rig extrinsic calibration using a motion capture system. 2018 5th IEEE Int. Work. Metrol. Aerosp. 2018:590595.

[14] Yang S, Zhang G. A review of interferometry for geometric measurement. Meas. Sci. Technol. 2018;29:102001.

[15] Lee D, Lee H, Yang S. Analysis of squareness measurement using a laser interferometer system. Int. J. Prec. Eng. Manuf. 2013;14:1839-1846.

[16] Schwenke H, Knapp W, Haitjema H, Weckenmann A, Schmitt R, Delbressine F. Geometric error measurement and compensation of machines-An update. Ann. CIRP 2008;57:660-675.

[17] Iñigo B, Ibabe A, Aguirre G, Urreta H. Analysis of laser tracker-based volumetric error. Metals 2019;9:757.

[18] Vahebi M, Arezoo B. Accuracy improvement of volumetric error modeling in CNC machine tools. Int. J. Adv. Manuf. Technol. 2018;95:2243-2257.

[19] Schwenke H, Schmitt R, Jatzkowski P, Warmann C. On-the-fly calibration of linear and rotary axes of machine tools and CMMs using a tracking interferometer. Ann. CIRP 2009;58:477-480.

[20] Schwenke H, Franke M, Hannaford J. Error mapping of CMMs and machine tools by a single tracking interferometer. Ann. CIRP 2005;54:475-478.

[21] Echerfaoui $Y$, Ouafi A El, Chebak A. Laser interferometer based measurement for positioning error compensation in cartesian multi-axis systems. J. Anal. Sci. Methods Instrum. 2017;07:75- 
92.

[22] Brauer-Burchardt C, Kuhmstedt P, Notni G. Improvement of measurement accuracy of optical 3D scanners by discrete systematic error estimation. IWCIA 2018 Comb. Image Anal. 2018;1:202-215.

[23] Gao W, Haitjema H, Fang FZ, Leach RK, Cheung CF, Savio E. On-machine and in-process surface metrology for precision manufacturing. Ann. CIRP 2019;68:843-866.

[24] Mendikute A, Yagüe-Fabra JA, Zatarain M, Bertelsen Á, Leizea I. Self-calibrated in-Process photogrammetry for large raw part measurement and alignment before machining. Sensors 2017;17:2066.

[25] Sims-Waterhouse D, Bointon P, Piano S, Leach RK. Experimental comparison of photogrammetry for additive manufactured parts with and without laser speckle projection. Proc. SPIE 2017;10329.

[26] Moulon P, Monasse P, Perrot R, Marlet R. OpenMVG : Open Multiple View Geometry. Work. Reprod. Res. Pattern Recognit. 2016;10214:60-74.

[27] Moulon, P Monasse P. Unordered feature tracking made fast and easy. Eur. Conf. Vis. Med. Prod. (London) 2012;5-6.

[28] Moulon P, Monasse P, Marlet R. Global fusion of relative motions for robust, accurate and scalable structure from motion. Proc. ICCV 2013;3248-3255.

[29] Verykokou S, loannidis C. A photogrammetry-based structure from motion algorithm using robust iterative bundle adjustment techniques. ISPRS Ann. 2018;IV:1-2.

[30] Westoby MJ, Brasington J, Glasser NF, Hambrey MJ, Reynolds JM. Structure-from-Motion photogrammetry: A low-cost, effective tool for geoscience applications. Geomorphology 2012;179:300-314.

[31] Percoco G, Modica F, Fanelli S. Image analysis for 3D micro-features: A new hybrid measurement method. Precis. Eng. 2017;48:123-132.

[32] Furukawa Y, Hernández C. Multi-view stereo: A tutorial. Found. Trends Comput. Graph. Vis. 2015;9:1-148.

[33] Zhang Z. A Flexible new technique for camera calibration. IEEE Trans. Pattern Anal. Mach. Intell. 2000;22:1330-1334.

[34] Sims-Waterhouse D, Piano S, Leach RK. Verification of micro-scale photogrammetry for smooth three-dimensional object measurement. Meas. Sci. Technol. 2017;28:055010.

[35] Flack D. CMM measurement stategies - Good practice guide No. 41. National Physical Laboratory; 2014.

[36] Zheng $Y$, Duan J. A geometric and kinematic error model and compensation of a 6-axis motorized optical fiber alignment stage. Adv. Mech. Eng. 2015;7:1-12.

[37] Eman KF, Wu BT, F. DM. A generalised geometric error model for multi-axis machines. Ann. CIRP 1987;36:253-256.

[38] Denavit J, Hartenberg RS. A kinematic notation for lower-pair mechanisms based on matrices. Trans ASME J. Appl. Mech. 1955;23:215-221.

[39] Hartley R, Zisserman A. Multiple view geometry in computer vision. Cambridge University Press; 2003.

[40] Slabaugh GG. Computing Euler angles from a rotation matrix. Tech. Rep. City Univ. London 1999;6:1-6.

[41] Hagan MT, Demuth HB, Beale MH, De Jesus O. Neural Network Design. PWS Publishing Co. 2014.

[42] Amidror I. Scattered data interpolation methods for electronic imaging systems: a survey. J. Electron. Imaging 2002;11:157.

[43] Lowe DG. Distinctive image features from scale-invariant keypoints. Int. J. Comput. Vis. 
2004;60:91-110.

[44] Cheng J, Leng $\mathrm{C}, \mathrm{Wu}$ J, Cui H, Lu H. Fast and accurate image matching with cascade hashing for 3D reconstruction. 2014 IEEE Conf. Comput. Vis. Pattern Recognit. 2014:1-8.

[45] Stew H, Engels C, Nist D. Recent developments on direct relative orientation. ISPRS J. Photogramm. Remote Sens. 2006;60:284-294.

[46] Ma KF, Huang GP, Xu HJ, Wang WF. Research on a precision and accuracy estimation method for close-range photogrammetry. Int. J. Pattern Recognit. Artif. Intell. 2019;33:1955002.

[47] Dai JS. Euler-Rodrigues formula variations, quaternion conjugation and intrinsic connections. Mech. Mach. Theory 2015;92:144-152.

[48] Isa MA, Sims-waterhouse D, Piano S, Leach R. Kinematic error analysis of stage tracking using stereo vision. Proc. ASPE (Pittsburg), 2019.

[49] VDI/VDE 2634 Part 3. Optical 3D-measuring systems - Multiple view systems based on area scanning. Gesellschaft Mess- und Automatisierungstechnik (Berlin). 2014. 